\title{
Editorial und Danksagung zum Wechsel in der Schriftleitung
}

\author{
Andrea Uber · Inga Lipp-Krebs
}

Online publiziert: 8. März 2019

(C) Springer-Verlag GmbH Deutschland, ein Teil von Springer Nature 2019

Liebe Leserinnen und Leser,

als zum Jahr 1999 mit dem 88. Band dieser Zeitschrift die Schriftleitung von Herrn Professor Dr. Dieter Farny, Köln an Herrn Professor Dr. J.-Matthias Graf von der Schulenburg, Hannover überging, verfasste dieser folgendes Editorial, dem wenig hinzuzufügen ist:

\section{Eine Zeitschrift mit langer Tradition erhält einen neuen Schriftleiter}

„1901 erschien der erste Band der Zeitschrift für die gesamte Versicherungswissenschaft. Am 25. Mai desselben Jahres wurde das Versicherungsaufsichtsgesetz für das Deutsche Reich eingeführt. Damit wurde nicht nur eine spezielle Marktordnung für die Versicherungswirtschaft geschaffen, sondern es etablierte sich ein neues wissenschaftliches Lehr- und Forschungsfeld, da sich spezielle juristische, wirtschaftliche, mathematische und medizinische Fragen in der Versicherungswirtschaft stellten, die auch mit den spezifischen rechtlichen Rahmenbedingungen dieser Sprache zu tun haben.

Der Name der Zeitschrift deutet darauf hin, dass nicht ein disziplinäres Publikationsorgan gewünscht war, sondern eine Zeitschrift, die offen ist für alle wissenschaftlichen Fragen, die in der Versicherungswirtschaft oder mit ihr aufgeworfen werden. Diesen Anspruch hat die Zeitschrift heute noch und er hat weiterhin seine Berechtigung, auch wenn zwischenzeitlich eine Vielzahl von disziplinären versicherungswissenschaftlichen Publikationsorganen für die Gebiete Versicherungswirtschaft, Versi-

\footnotetext{
A. Uber · I. Lipp-Krebs $(\bowtie)$

Deutscher Verein für Versicherungswissenschaft e. V., Rheinstr. 45-46, 12161 Berlin, Deutschland E-Mail: inga.lipp-krebs@dvfvw.de
} 
cherungsrecht, Versicherungsmedizin und Versicherungsmathematik gegründet wurden. Es wird eine besondere Herausforderung (...) sein, die Interdisziplinarität der ZVersWiss zu stärken (...).

Die ZVersWiss muss und wird für die Behandlung neuer Fragestellungen immer offen sein. Mein Vorgänger Dieter Farny, der dieses Amt seinerzeit von Reimer Schmidt übernahm, hat die Zeitschrift in diesem Geiste der Interdisziplinarität und Wissenschaftlichkeit geleitet (...).

$\mathrm{Ob}$ der Anspruch einer hochkarätigen und lesenswerten wissenschaftlichen Zeitung eingelöst werden kann, liegt primär an den potentiellen Autoren, die unserer Zeitschrift ihre Manuskripte anvertrauen. Da es mittlerweile viele versicherungswissenschaftliche Publikationsorgane im In- und Ausland gibt, besteht auch hier Wettbewerb, dem sich die ZVersWiss stellen muss. So wird die Zeitschrift auch für englischsprachige Publikationen offen sein (...)“.

Nunmehr ist es am Vorstandsvorsitzenden des Deutschen Vereins für Versicherungswissenschaft e. V. Herrn Professor Dr. Wolfgang Weiler dem zum Band 108 (2019) der Zeitschrift scheidenden Schriftleiter Herrn Professor von der Schulenburg und seiner Schriftleitungsassistenz Frau Dr. Lohse für ihr Engagement zu danken.

Außerdem gebührt der Dank allen Autoren für ihre Manuskripteinreichungen sowie den aufgeführten Bereichsschriftleitern und Gutachtern des letzten Jahres: Christine Arentz, Christian Armbrüster, Tobias Basse, Stephan Becher, Meik Friedrich, Peter Grundke, Helmut Gründl, Tim Linderkamp, Ute Lohse, Ralf Lohse, Angelika May, Kay Peters, Sebastian Reddemann, Miguel Rodriguez, Christoph Schwarzbach, Heinz-Dietrich Steinmeyer, Manfred Wandt und Christoph Wegener.

Im Kreis der Bereichsschriftleiter 2019 bestehend aus Professor Dr. Manfred Wandt, Frankfurt am Main (Versicherungsrecht), Professorin Dr. Angelika May, Oldenburg (Versicherungsmathematik) und Privatdozent Dr. med. habil. Stephan Becher, Köln (Versicherungsmedizin) dürfen wir Professor Dr. Heinrich R. Schradin, Köln, als neuen verantwortlichen Bereichsschriftleiter für den Bereich Versicherungsökonomie vorstellen. Damit entfällt die Position des Hauptschriftleiters.

Last but not least möchten wir alle Autorinnen und Autoren bitten, ihre Manuskripte fortan unter redaktion.zverswiss@dvfvw.de einzureichen.

Dr. Andrea Uber (Geschäftsführung)

Inga Lipp-Krebs (Schriftleitungsassistenz) 